\title{
SIMULAÇÃO DA DESTILAÇÃO DO PRODUTO OBTIDO A PARTIR DO CRAQUEAMENTO DE ÓLEO DE PALMA EMPREGANDO O SIMULADOR ASPEN HYSYS.
}

\author{
E. C. $\operatorname{COSTA}^{1}$, A. K. TAKAKURA ${ }^{1}$, N. T. MACHADO ${ }^{1}$. \\ ${ }^{1}$ Universidade Federal do Pará, Programa de Pós-Graduação em Engenharia de Recursos Naturais da \\ Amazônia. \\ E-mail para contato: elineia_castro@yahoo.com.br
}

\begin{abstract}
RESUMO - A utilização de simuladores de processo contribui de forma significativa para o desenvolvimento e otimização de processos industriais. Neste contexto, este trabalho tem como objetivo realizar simulações utilizando o pacote computacional comercial Aspen Hysys ${ }^{\circledR}$, a fim de analisar os parâmetros operacionais que influenciam a separação dos componentes indesejáveis no produto obtido a partir do craqueamento de óleo vegetal. As simulações foram realizadas variando-se a razão de refluxo e o número de pratos de uma coluna de destilação, mantendo-se constantes a pressão $(101,3 \mathrm{kPa})$, a temperatura $\left(70^{\circ} \mathrm{C}\right)$ e a composição da corrente de entrada do sistema multicomponente. A composição da corrente de entrada (hidrocarbonetos na faixa do querosene) foi retirada de dados da literatura obtidos experimentalmente a partir de uma unidade de craqueamento onde se utilizou óleo de palma para obter biocombustível.
\end{abstract}

\section{INTRODUÇÃO}

$\mathrm{Na}$ indústria química o processo de separação mais amplamente utilizado é a destilação. $\mathrm{Na}$ destilação, uma fase vapor entra em contato com uma fase líquida, e há transferência de massa entre as fases. Uma das mais importantes características da destilação é que esta não requer a adição de nenhuma substância para efetivar a separação, ela está baseada no equilíbrio de fases (Duarte, 2006). $\mathrm{Na}$ indústria do petróleo, a destilação é o processo de separação mais utilizado, para separar o óleo cru em diversas frações e posteriormente para obter produtos intermediários. Na produção de biocombustível a destilação também se mostra extremamente importante. No craqueamento de óleos vegetais para a obtenção de biocombustível a destilação pode ser utilizada para separar as frações de produtos e posteriormente para auxiliar no processo de purificação. Lhamas (2013) destilou o produto liquido obtido a partir do craqueamento de óleo de palma e buriti e obteve produtos compostos majoritariamente por hidrocarbonetos na faixa correspondente à gasolina, diesel e querosene de petróleo. Nestes processos as frações obtidas, geralmente, necessitam ainda de etapas adicionais para a purificação, e a destilação pode ser novamente utilizada para separar compostos indesejáveis na mistura de produtos, baseando-se na diferença entre os pontos de ebulição dos componentes.

Neste contexto, o uso de simuladores de processos se mostra de extrema importância, pois tais ferramentas são capazes de descrever o comportamento de processos industriais (dentre eles a 


\section{9 a 22 de outubro de 2014 \\ Florianópolis/SC}

purificação de biocombustíveis) com aceitável precisão, possibilitando e contribuindo para a otimização desses processos. Neste trabalho foram elaboradas plantas simplificadas de destilação visando à retirada dos compostos oxigenados e hidrocarbonetos aromáticos de uma mistura similar ao querosene de petróleo, obtida após a purificação do produto resultante do craqueamento de óleo de palma realizado por Mota (2013). Empregando o simulador de processos comercial Aspen Hysys o trabalho tem como um dos objetivos principais investigar as variações nas frações de oxigenados e aromáticos nas correntes de saída do destilador, frente à variação da razão de refluxo e número de estágios da coluna. Segundo Farah (2012) o QAV-1 (querosene de aviação) apresenta predominância de hidrocarbonetos parafínicos, compostos mais desejáveis para se obter chamas limpas e sem fuligem, contendo também hidrocarbonetos aromáticos que apresentam as piores características de combustão, pois, provocam aumento da radiação na câmara de combustão, assim como a formação de fuligem, sendo necessária sua remoção. Quanto à presença de oxigenados, estes favorecem o aumento da acidez, corrosividade, formação de gomas e odor, sendo também, nesse caso, necessária a remoção de tais compostos (Lhamas, 2013).

\section{SIMULAÇÃO DE PROCESSOS}

\subsection{Modelos Termodinâmicos}

O sucesso da modelagem de processos depende da precisão da descrição das propriedades termodinâmicas e das condições de equilíbrio de fases. O simulador ASPEN HYSYS possui diversas opções de modelos termodinâmicos para descrever o equilíbrio de fases da maioria dos processos aplicados na indústria química. Estes modelos termodinâmicos pretendem interpretar e predizer o comportamento físico-químico da matéria em equilíbrio (Takakura, 2009). O cálculo do equilíbrio de fases pode ser baseado na aplicação de equações de estado (EDE). Assim, uma única equação é usada para representar todas as fases fluidas (Teribeli, et al. 2006).

As equações de estado cúbicas mais amplamente utilizadas na indústria são as de SoaveRedlich-Kwong (SRK) e Peng-Robinson (PR) e suas modificações. O HYSYS atualmente oferece as EDE's de (PR) e (SRK) melhoradas. Além de várias modificações destes pacotes de propriedades. Entre elas, a EDE de Peng-Robinson suporta a mais ampla gama de condições de funcionamento e a maior variedade de sistemas. As EDE's PR e SRK podem gerar todo o equilíbrio e propriedades termodinâmicas necessárias diretamente, só necessitando das informações das propriedades críticas e fatores acêntricos dos compostos puros. Embora as formas destas EDE's sejam comumente encontradas em outros simuladores comerciais, elas foram significativamente reforçadas no simulador para alargar a sua gama de aplicabilidade (Aspentech, 2000). A equação 1 apresenta a forma da equação proposta por Peng-Robson (1976).

$$
P=\frac{R T}{v-b}-\frac{a(T)}{v(v+b)+b(v-b)}
$$

A equação 2 , representa a forma da dependência do parâmetro $a$, do termo atrativo, com a temperatura proposta por Soave (1972). Esta proposta proporcionou uma descrição mais adequada do comportamento da fase líquida e da pressão de vapor. A forma de dependência do parâmetro $a$ para a equação de Peng-Robinson é a mesma proposta por Soave (1972). 


$$
\begin{aligned}
& a(T)=a\left(T_{c}\right) \alpha\left(T_{r}, \omega\right) \\
& \alpha\left(T_{r}, \omega\right)=\left\{1+k_{m}\left[1-\left(T_{r}\right)^{1 / 2}\right]\right\}^{2} \\
& k_{m}=0,37464+1,54226 \omega-0,26992 \omega^{2}
\end{aligned}
$$

As Equações 4 foi determinada para a equação PR. O simulador Aspen-Hysys também disponibiliza a opção de uso da Equação 5 para a equação de PR quando o fator acêntrico é maior que 0,49 (Teribeli, et al. 2006).

$$
k_{m}=0,379642+1,48503 \omega_{i}-0,16442376 \omega_{i}^{2}+1,016666 \omega_{i}^{3}
$$

As relações que expressam a dependência com a composição dos parâmetros $a$ e $b$ da equação de estado, são chamadas de regras de mistura. As regras de mistura de van der Waals, com as regras combinadas para dois parâmetros de interação binária, são utilizadas no Aspen Hysys e são dadas pelas equações 6 e 7. Os parâmetros kij são parâmetros de interação binária, ajustáveis a dados experimentais de equilíbrio. Neste trabalho foi utilizada a base de parâmetros de interação disponíveis no Aspen Hysys.

$$
\begin{aligned}
& a=\sum_{i=1}^{N} \sum_{j=1}^{N} x_{i} x_{j}\left(a_{i} a_{j}\right)^{0,5}\left(1-k_{i j}\right) \\
& b=\sum_{i=1}^{N} x_{i} b_{i}
\end{aligned}
$$

Como visto anteriormente a simulação utilizando o Aspen Hysys requer algumas informações sobre as substancias envolvidas na simulação. As propriedades críticas e o fator acêntrico dos componentes utilizados são informações indispensáveis à simulação. Diante disto, neste trabalho foi utilizada a metodologia de contribuição de grupos proposta por Constantinou e Gani (1994) e Constantinou et al. (1995) para predizer as propriedades termofísicas e o fator acêntrico dos componentes presentes nas mistura utilizada. Estes métodos foram desenvolvidos para melhorar a precisão da predição considerando dois tipos de grupos para dividir as moléculas.

\subsection{Simulação da destilação}

O projeto foi concebido segundo algumas premissas e considerações: o modelo termodinâmico selecionado para realizar as simulações foi o proposto por Peng-Robinson (1976); a pressão de operação, assim como, a temperatura da corrente de entrada foram as mesmas para todas as simulações; a composição da corrente de alimentação utilizada foi a obtida experimentalmente por Mota (2013).

Mota (2013) realizou o craqueamento de óleo de palma e após algumas etapas de separação dos componentes do produto obtido com o craqueamento, obteve como um dos produtos um biocombustível na faixa de destilação de $175-235^{\circ} \mathrm{C}$. Através da análise desse biocombustível, Mota (2013) afirmou que a faixa dos hidrocarbonetos presentes no biocombustível obtido entre 175 $235^{\circ} \mathrm{C}$, continha hidrocarbonetos entre $\mathrm{C}_{8}$ a $\mathrm{C}_{17}$, sendo preponderante a faixa de hidrocarbonetos $\mathrm{C}_{11}$ $\mathrm{a}_{12}$. Desta forma, o autor inferiu que, o biocombustível se aproximava da faixa de hidrocarbonetos presentes no querosene derivado do petróleo, apesar de apresentar um percentual de hidrocarbonetos 
na faixa da gasolina. O bio-querosene; obtido por Mota (2013) corresponde a um biocombustível composto de hidrocarbonetos aromáticos, parafínicos normais, naftênicos e olefínicos, além de compostos oxigenados. A Tabela 1 apresenta a composição do biocombustível obtido por Mota (2013) referente ao teor de oxigenados e hidrocarbonetos. Salienta-se que no simulador foi cadastrado cada componente separadamente, e utilizada a composição de acordo com a Tabela 2, porém, visando simplificar a análise da simulação as frações de hidrocarbonetos não-aromáticos foram somadas, bem como, as de todos os oxigenados e hidrocarbonetos aromáticos.

Tabela 1 - Composição utilizada nas simulações

\begin{tabular}{cc}
\hline Componentes & Porcentagem em massa (\%) \\
\hline Hidrocarbonetos Aromáticos & 7,04 \\
Hidrocarbonetos Não aromáticos & 79,33 \\
Oxigenados & 13,63 \\
\hline
\end{tabular}

Tabela 2 - Composição cadastrada no simulador

\begin{tabular}{ccc}
\hline Fórmula Molecular & Compostos & Fração Mássica \\
\hline $\mathrm{C}_{8} \mathrm{H}_{10}$ & Etilbenzeno & 0,015659955 \\
$\mathrm{C}_{10} \mathrm{H}_{18}$ & ciclohexeno, 1-butil- & 0,036200936 \\
$\mathrm{C}_{11} \mathrm{H}_{18} \mathrm{O}$ & 1-propanona, 1 - (3-ciclo-hexen-1-il) -2,2-dimetil- & 0,011897498 \\
$\mathrm{C}_{9} \mathrm{H}_{16}$ & 1H-indeno, octa-hidro-cis- & 0,009151922 \\
$\mathrm{C}_{10} \mathrm{H}_{18}$ & 9-metilbiciclo [3.3.1] nonano & 0,00823673 \\
$\mathrm{C}_{12} \mathrm{H}_{26}$ & Dodecano & 0,131380923 \\
$\mathrm{C}_{7} \mathrm{H}_{14} \mathrm{O}$ & 2-heptanona & 0,045657921 \\
$\mathrm{C}_{11} \mathrm{H}_{22}$ & 1-undeceno & 0,184767134 \\
$\mathrm{C}_{16} \mathrm{H}_{30}$ & 1-hexadecino & 0,035184055 \\
$\mathrm{C}_{11} \mathrm{H}_{22}$ & ciclopropano, nonil- & 0,051149075 \\
$\mathrm{C}_{12} \mathrm{H}_{24}$ & benzeno, 1-etil-2-metil- & 0,056131788 \\
$\mathrm{C}_{9} \mathrm{H}_{12}$ & ciclopentano, 1-hexil-3-metil- & 0,034472239 \\
$\mathrm{C}_{12} \mathrm{H}_{24}$ & 1H-indeno, octa-hidro-5-metil- & 0,014236323 \\
$\mathrm{C}_{12} \mathrm{H}_{24}$ & Ciclohexano-ethanol & 0,010372178 \\
$\mathrm{C}_{8} \mathrm{H}_{16} \mathrm{O}$ & ciclopenteno,1-hexil- & 0,008033354 \\
$\mathrm{C}_{11} \mathrm{H}_{13} \mathrm{NO}$ & 1,4-undecadiene, (E) - & 0,016066707 \\
$\mathrm{C}_{11} \mathrm{H}_{20}$ & 1-dodeceno & 0,006813097 \\
$\mathrm{C}_{11} \mathrm{H}_{20}$ & ciclohexeno, 1-pentil- & 0,013626195 \\
$\mathrm{C}_{12} \mathrm{H}_{24}$ & 2-dodeceno, (Z) - & 0,0950783 \\
$\mathrm{C}_{11} \mathrm{H}_{20}$ & benzeno, butil- & 0,007321538 \\
$\mathrm{C}_{12} \mathrm{H}_{24}$ & benzeno, 1-metil-2-propil- & 0,007524914 \\
$\mathrm{C}_{10} \mathrm{H}_{14}$ & 2-pentadecano & 0,008135042 \\
$\mathrm{C}_{10} \mathrm{H}_{14}$ & $\mathrm{C}_{6} \mathrm{H}_{12} \mathrm{O}_{2}$ & 0,006711409 \\
$\mathrm{C}_{15} \mathrm{H}_{32}$ & & 0,015151515 \\
& & 0,047081554 \\
\hline
\end{tabular}


Tabela 3 - Composição cadastrada no simulador (continuação)

\begin{tabular}{c}
\hline Fórmula Molec \\
\hline $\mathrm{C}_{9} \mathrm{H}_{10}$ \\
$\mathrm{C}_{14} \mathrm{H}_{28}$ \\
$\mathrm{C}_{9} \mathrm{H}_{18} \mathrm{O}$ \\
$\mathrm{C}_{14} \mathrm{H}_{30}$ \\
$\mathrm{C}_{10} \mathrm{H}_{20} \mathrm{O}$ \\
$\mathrm{C}_{17} \mathrm{H}_{36}$ \\
$\mathrm{C}_{15} \mathrm{H}_{30}$ \\
\hline
\end{tabular}

\section{Compostos}

Indano

1-tetradeceno

2-nonanona

Tetradecano

2-decanona

Heptadecano

1-pentadeceno
Fração Mássica

0,006609721

0,055725036

0,016473459

0,012711003

0,008440106

0,006914785

0,017083588

As condições utilizadas na simulação podem ser visualizadas na tabela 3.

Tabela 4 - Condições Utilizadas na Simulação

\begin{tabular}{|c|c|c|c|c|c|c|}
\hline Número de Estágios & \multicolumn{3}{|c|}{11} & \multicolumn{3}{|c|}{21} \\
\hline Razão de Refluxo & 0,5 & 0,75 & 1 & 0,5 & 0,75 & 1 \\
\hline $\mathrm{T}_{\text {alimentacão }}\left({ }^{\circ} \mathrm{C}\right)$ & 70 & 70 & 70 & 70 & 70 & 70 \\
\hline $\mathrm{P}$ (bar) & 1,013 & 1,013 & 1,013 & 1,013 & 1,013 & 1,013 \\
\hline Fluxo Molar alimentacão $(\mathrm{kmol} / \mathrm{h})$ & 100 & 100 & 100 & 100 & 100 & 100 \\
\hline
\end{tabular}

A Figura 1(a) apresenta o equipamento da planta de destilação e a Figura1(b) o mesmo equipamento de forma mais detalhada pelo simulador.

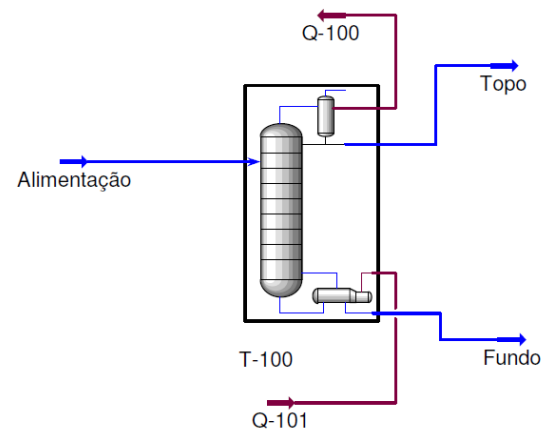

(a)

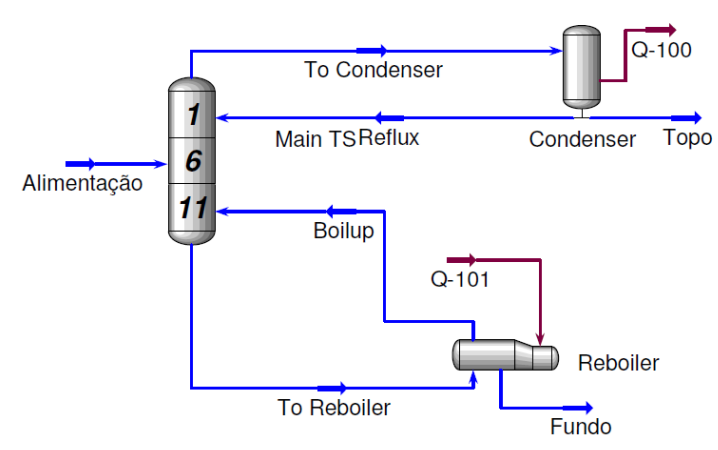

(b)

Figura 1- Equipamento da Planta de Destilação.

\section{RESULTADOS E DISCUSSÕES}

As tabelas 4 e 5 apresentam os valores referentes à temperatura, fluxos mássico e molar, e frações das correntes de produtos da simulação.

Tabela 5 - Resultados das Simulações com Coluna de Destilação de 11 Estágios

\begin{tabular}{ccccccc}
\hline 11 Estágios & \multicolumn{3}{c}{ Corrente de Topo } & \multicolumn{3}{c}{ Corrente de Fundo } \\
\hline Razão de Refluxo & 0,5 & 0,75 & 1 & 0,5 & 0,75 & 1 \\
\hline $\mathrm{T}\left({ }^{\circ} \mathrm{C}\right)$ & 65,92 & 62,80 & 64,17 & 210,87 & 209,77 & 210,41 \\
\hline
\end{tabular}


Tabela 6 - Resultados das Simulações com Coluna de Destilação de 11 Estágios (cont.)

\section{Estágios}

Fluxo Mássico $(\mathrm{kg} / \mathrm{h})$

Fluxo Molar (kmol/h)

Fração Mássica

Fração Molar
Corrente de Topo

$5730,26 \quad 5249,88$

$41,7999 \quad 38,6667$

5450,56

39,9999

0,2067

0,5491

0,2156

0,2365

0,5021

0,2380
Corrente de Fundo

\begin{tabular}{ccc}
10010,58 & 10490,96 & 10290,28 \\
58,2000 & 61,3333 & 60,0000 \\
0,0000 & 0,0000 & 0,0000 \\
0,8713 & 0,9415 & 0,9431 \\
0,0547 & 0,0546 & 0,0538 \\
0,0000 & 0,0000 & 0,0000 \\
0,8721 & 0,9272 & 0,9292 \\
0,0680 & 0,0688 & 0,0675 \\
\hline
\end{tabular}

*A - Hidrocarbonetos Aromáticos; B - Hidrocarbonetos não aromáticos; C - Compostos Oxigenados.

Tabela 7 - Resultados das Simulações com Coluna de Destilação de 21 Estágios

\begin{tabular}{cccccccc}
\hline 21 Estágios & \multicolumn{3}{c}{ Corrente de Topo } & \multicolumn{3}{c}{ Corrente de Fundo } \\
\hline Razão de Refluxo & & 0,5 & 0,75 & 1 & 0,5 & 0,75 & 1 \\
\hline T $\left({ }^{\circ} \mathrm{C}\right)$ & & 65,97 & 62,84 & 64,19 & 211,47 & 210,31 & 210,92 \\
Fluxo Mássico $(\mathrm{kg} / \mathrm{h})$ & & 5717,20 & 5239,56 & 5440,23 & 10023,63 & 10501,28 & 10300,61 \\
Fluxo Molar $(\mathrm{kmol} / \mathrm{h})$ & & 41,7999 & 38,6670 & 40,0002 & 58,2001 & 61,3330 & 59,9998 \\
& $\mathrm{~A}$ & 0,1971 & 0,2151 & 0,2071 & 0,0000 & 0,0000 & 0,0000 \\
Fração Mássica & $\mathrm{B}$ & 0,5618 & 0,5349 & 0,5468 & 0,9463 & 0,9423 & 0,9440 \\
& $\mathrm{C}$ & 0,2109 & 0,2177 & 0,2138 & 0,0522 & 0,0560 & 0,0549 \\
& $\mathrm{~A}$ & 0,2263 & 0,2446 & 0,2365 & 0,0000 & 0,0000 & 0,0000 \\
Fração Molar & $\mathrm{B}$ & 0,5149 & 0,4892 & 0,5005 & 0,9333 & 0,9281 & 0,9303 \\
& $\mathrm{C}$ & 0,2340 & 0,2398 & 0,2366 & 0,0652 & 0,0701 & 0,0685 \\
\hline
\end{tabular}

*A - Hidrocarbonetos Aromáticos; B - Hidrocarbonetos não aromáticos; C - Compostos Oxigenados.

A Figura 2 ilustra o gráfico das frações mássicas de hidrocarbonetos não aromáticos nas correntes de topo e fundo das plantas para cada razão de refluxo estudada.

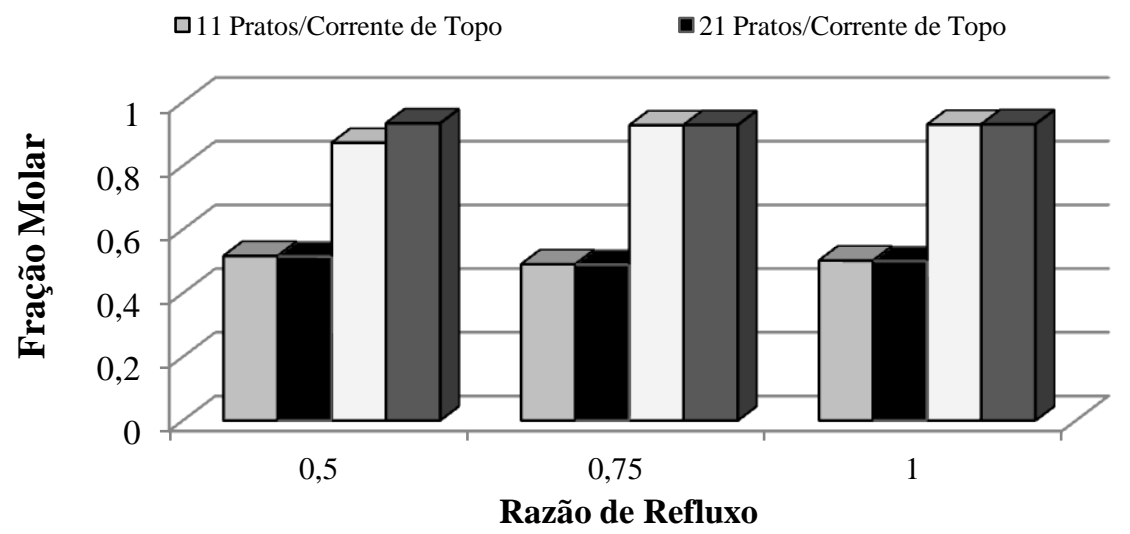

Figura 2 - Gráfico das Frações Molares de Hidrocarbonetos Não Aromáticos.

No que se refere à variação do número de estágios da coluna de destilação, o efeito mais 
significativo foi observado na razão de refluxo de 0,5 , onde a fração de hidrocarbonetos não aromáticos aumentou cerca de $6 \%$ na corrente de fundo com o aumento do número de estágios. A variação das frações com o número de estágios das plantas à razão 0,75 e 1, não apresentou valores muito significantes. No que se refere à variação da razão de refluxo podemos observar que o aumento de 0,5 para 0,75 forneceu um aumento na fração de hidrocarbonetos não aromáticos em torno de $5,5 \%$ na corrente de fundo para a coluna de 11 estágios, o mesmo comportamento não é observado para a de 21 estágios onde a variação da fração de hidrocarbonetos é inversa e bem menos sensível. As variações com o aumento da razão de 0,75 para 1, também não se mostraram muito significativas.

A figura 3 ilustra a variação da composição da fase vapor ao longo dos estágios da coluna para a planta cujo destilador operou com 11 estágios e à razão de refluxo igual a 0,5.

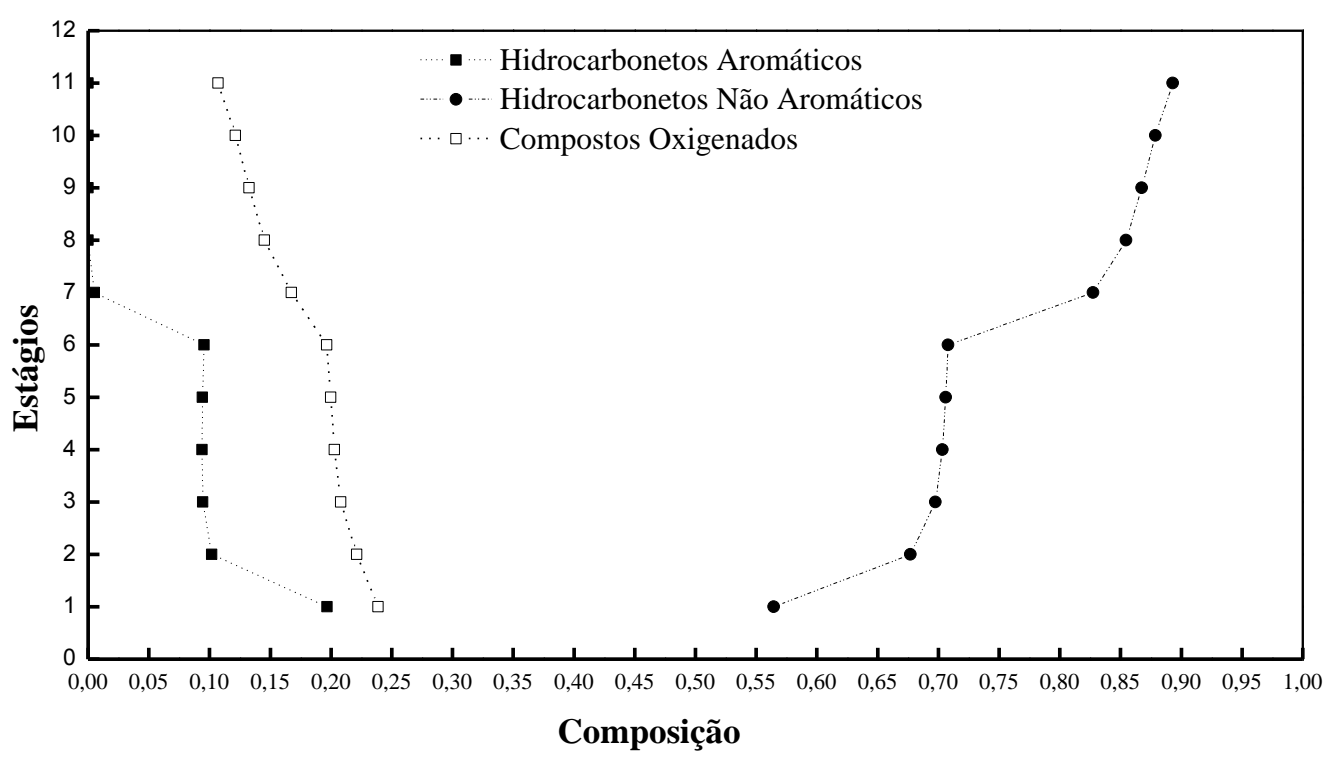

Figura 3 - Composição ao Longo dos Estágios.

Através do gráfico é possível observar o enriquecimento de hidrocarbonetos aromáticos e compostos oxigenados da entrada da coluna (estágio 6) em direção ao topo (prato 1), e o comportamento inverso no que se refere a fração dos demais componentes da mistura. A Fração de hidrocarbonetos não aromáticos é maior no estágio 11.

\section{CONCLUSÃO}

Os resultados da simulação simplificada da destilação do produto (similar ao querosene de petróleo) obtido através a purificação da mistura resultante do craqueamento de óleo de palma, demonstraram bons resultados no que se refere à retirada de hidrocarbonetos aromáticos e compostos oxigenados, prejudiciais à qualidade do "bioquerosene". A variação do número de estágios e da razão de refluxo da coluna de destilação não mostrou ter influência muito significativa na retirada de aromáticos, visto que, a fração destes se manteve praticamente constante na corrente de topo para todas as plantas. Quanto aos oxigenados a planta cuja coluna operou com 11 estágios e razão de 
refluxo igual a 0,5, apresentou maior concentração de oxigenados na corrente de topo.

\section{REFERENCIAS}

ASPENTECH,Thermodynamics and HYSYS, 2000.

CONSTANTINOU, L.; GANI, R. New group contribution method for estimating properties of pure compounds. American Institute of Chemical Engineers Journal. v. 40, p. 1697-1710, 1994.

CONSTANTINOU, L.; GANI, R.; O'CONNELL, J. P. Estimation of the acentric factor and the liquid molar volume at $298 \mathrm{~K}$ using a new group contribution method. Fluid Phase Equilibria. v. 103, p. 11-22, 1995.

DUARTE, L. A. Modelagem e Simulação de uma Unidade de Separação dos Componentes provenientes de um Reator FT (Fischer-Tropsch) através do Simulador HYSYS ${ }^{\mathrm{TM}}$. 2006. 58 f. Monografia (Bacharelado em Engenharia Química) - Universidade Federal do Rio Grande do Norte. Natal - RN, 2006.

FARAH, M. A. Petróleo e seus derivados: definição, constituição, aplicação, especificações, características de qualidade. Rio de Janeiro: LTC, 2012.

LHAMAS, D. E. L. Estudo do Processo de Craqueamento Termocatalítico do Óleo de Palma (Elaeis guineensis) e do Óleo de Buriti (Mauritia flexuosa L.) para Produção de Biocombustível. 2013. 216 f. Tese (Doutorado em Engenharia de Recursos Naturais) - Universidade Federal do Pará. Belém - PA, 2013.

MOTA, S. A. P.Craqueamento termo-catalítico de óleos vegetais em diferentes escalas de produção. 2013. 332 f. Tese (Doutorado em Engenharia de Recursos Naturais)- Universidade Federal do Pará. Belém, Pará, 2013.

PENG, D.Y.; ROBINSON, D.B. A New Two-Constant Equation of State. Industrial and Engineering Chemical Fundamental 15: 59-64, 1976.

SOAVE, G. Equilibrium Constants from a Modified Redlich-Kwong Equation of State. Chemical Engineering Science v. 27, p.1192-1203, 1972.

TAKAKURA, A. K. Simulação do Processo Contínuo de Produção e Purificação de Biodiesel de Óleo d Palma Bruto. 2009. 96 f. Dissertação (Mestrado em Engenharia Química) - Universidade Federal do Pará. Belém - PA, 2009.

TERIBELE, T.; SANTOS, J. L.; ARAÚJO, M. E. Simulação De Uma Unidade De Turbo-Expansão De Gás Natural Empregando Diferentes Equações De Estado. In: Cobeq 2006 - XVI Congresso Brasileiro de Engenharia Química e III Congresso Brasileiro de Termodinâmica Aplicada, 2006, Santos. 